\title{
Characterization of Echinostoma cinetorchis endoribonuclease, RNase $\mathrm{H}$
}

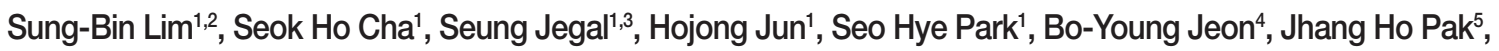 \\ Young Yil Bakh ${ }^{6}$, Tong-Soo Kim ${ }^{1, *}$, Hyeong-Woo Lee ${ }^{7, *}$ \\ 'Department of Tropical Medicine and Parasitology, Inha University School of Medicine, Incheon 22212, Korea; '2Department of Bioscience, Dongkuk \\ University, Seoul 04620, Korea; ${ }^{3}$ Department of Infectious Diseases Diagnosis, Incheon Institute of Public Health and Environment, Incheon 22320 , \\ Korea; ${ }^{4}$ Department of Biomedical Laboratory Science, College of Health Sciences, Yonsei University, School of Public Health, Wonju 26493, Korea; \\ ${ }^{5}$ Department of Convergence Medicine, University of Ulsan, College of Medicine, Asan Institute for Life Sciences, Asan Medical Center, Seoul \\ 05505, Korea; ${ }^{6}$ Department of Biotechnology, Konkuk University, Chungju 27478, Korea; ${ }^{7}$ Institute of Research and Development, Scorpiogen Co., \\ Anseong 17579, Korea
}

\begin{abstract}
Echinostoma cinetorchis is an oriental intestinal fluke causing significant pathological damage to the small intestine. The aim of this study was to determine a full-length cDNA sequence of $E$. cinetorchis endoribonuclease (RNase $\mathrm{H}$; EcRNH) and to elucidate its molecular biological characters. EcRNH consisted of 308 amino acids and showed low similarity to endoribonucleases of other parasites $(<40 \%)$. EcRNH had an active site centered on a putative DDEED motif instead of DEDD conserved in other species. A recombinant EcRNH produced as a soluble form in Escherichia coli showed enzymatic activity to cleave the 3'-O-P bond of RNA in a DNA-RNA duplex, producing 3'-hydroxyl and 5'-phosphate. These findings may contribute to develop antisense oligonucleotides which could damage echinostomes and other flukes.
\end{abstract}

Key words: Echinostoma cinetorchis, endoribonuclease, recombinant protein, localization, RNase $\mathrm{H}$

Echinostomes are intestinal flukes that are widespread in Southeast Asia. Four species are prevalent in Korea; Echinostoma hortense, Echinostoma cinetorchis, Echinochasmus japonicus, and Acanthoparyphium tyosenense. Infections by echinostomes are associated with more severe symptoms than those due to other intestinal flukes, because they are larger than other flukes and adults have head crowns and collar spines that can cause serious stomach damage [1].

This fluke has a head crown with 37-38 collar spines, including 5 spines on both ends. It also has testes that are not constant in number and are not fixed in a single location [2]. Flukes were studied mainly by Japanese researchers until 1960, with a focus on human infections [3-5], intermediate hosts, reservoir hosts, and biological and epidemiological characteristics [1]. As a result of these studies, large shellfish, frogs, tadpoles, and loaches were found to act as the first intermediate

- Received 16 September 2016, revised 27 July 2017, accepted 2 August 2017.

*Corresponding authors (tongsookim@inha.ac.kr; rainlee67@naver.com) (c) 2017, Korean Society for Parasitology and Tropical Medicine

This is an Open Access article distributed under the terms of the Creative Commons Attribution Non-Commercial License (http://creativecommons.org/licenses/by-nc/4.0) which permits unrestricted non-commercial use, distribution, and reproduction in any medium, provided the original work is properly cited. hosts $[1,6,7]$. The fluke has been found to be widespread in Rattus norvegicus, which lived in inland of Korea during nationwide surveys on infection of reservoir hosts with these flukes [8].

Recent researches on parasites have focused on developing vaccines and chemotherapeutic drugs by elucidating the pathological and biochemical characteristics of specific molecules, especially proteinases and oligonucleotide targeting. However, there was been very little biochemical and molecular biological research on E. cinetorchis, as the majority of studies on this species have focused on the life cycle and intermediate host exploration.

Therefore, we cloned and examined the characteristics of an endoribonuclease of $E$. cinetorchis.

Endoribonuclease can cut single-stranded and doublestranded RNA. The proteins include RNase III, RNase A, RNase T1, RNase H, RNase P, and RNA-induced silencing complex. Turnover of mRNA is very important for gene regulation. Exonucleases are known to be involved in mRNA degradation that controls gene expression and has $5^{\prime}-3^{\prime}$ exonucleolytic and $3^{\prime}-5^{\prime}$ exonucleolytic activity. However, little is known about endoribonucleases that degrade RNA at internal sequences [9]. Re- 


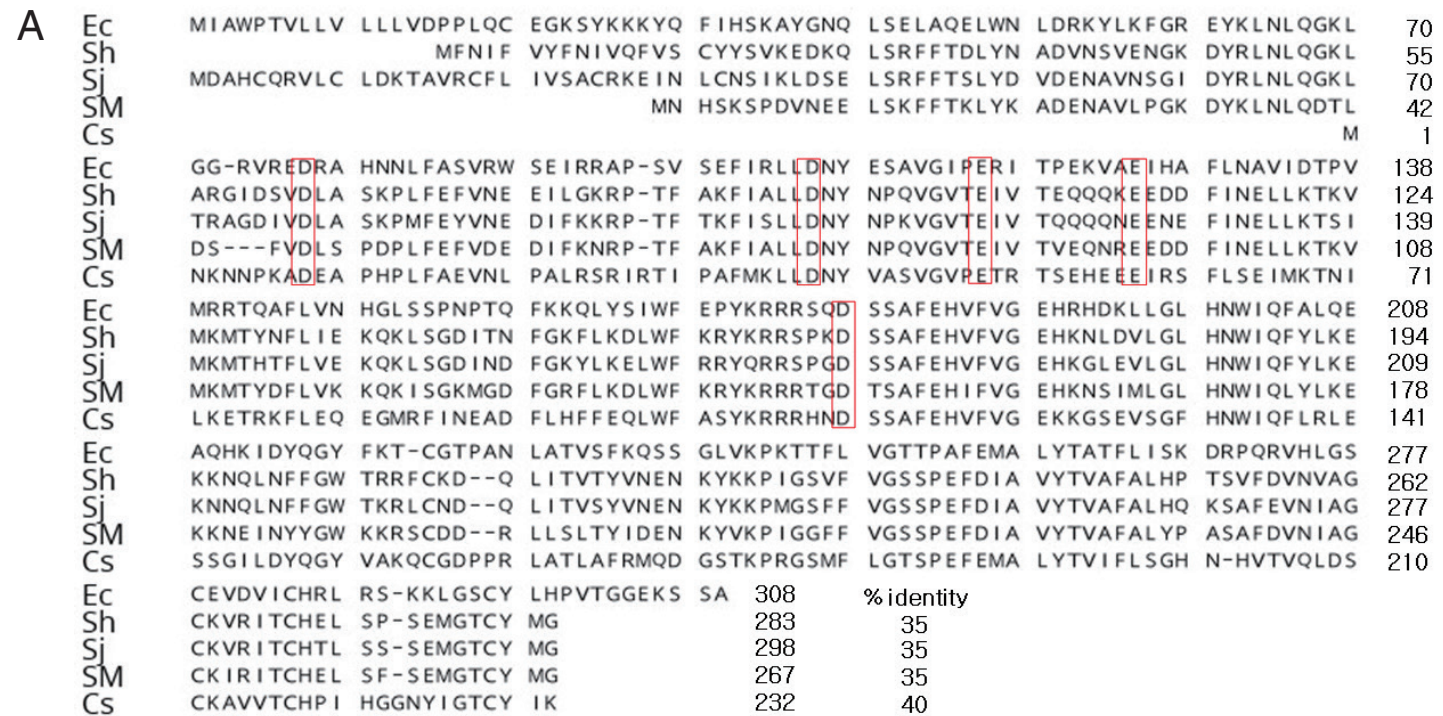

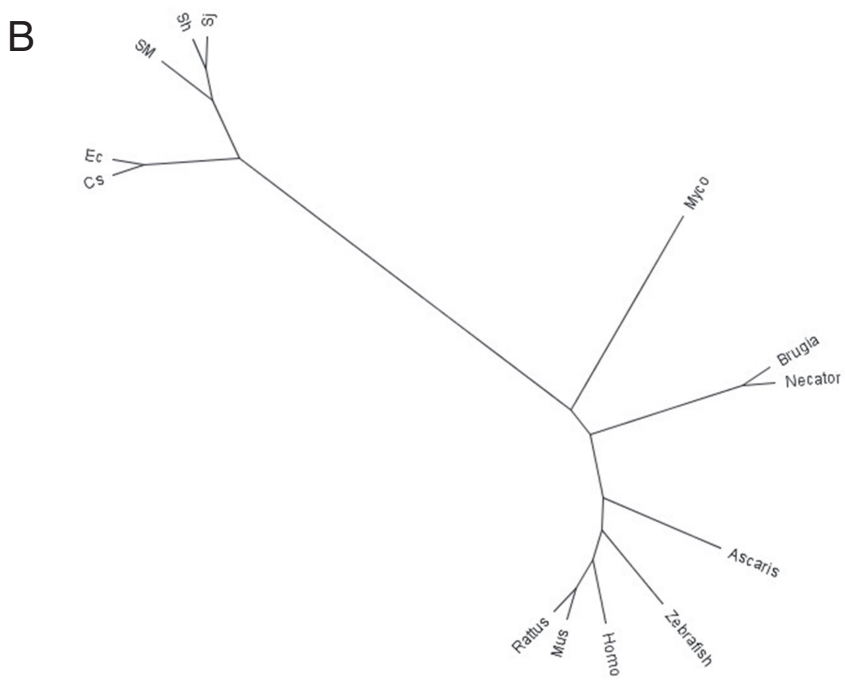

Fig. 1. Multiple alignment of amino acid sequence of Echinostoma cinetorchis with homologs from other species. (A) Putative active sites (red boxes) of aligned RNase H. (B) Phylogenic tree of RNase H of several species. GenBank accession nos.: Sh (Schistosoma haematobium, KGB32409.1), Sj (Schistosoma japonicum, AAW25442.1), Sm (Schistosoma mansoni, CCD59795.1), Cs (Clonorchis sinensis, GAA56298.1), Ec (E. cinetorchis of Korean isolate), Myco (Mycobacterium tuberculosis, AGQ35548.1), Rat (Rattus norvegicus, AAH91209.1), Mus (Mus musculus, AAH19411.1), Homo (Homo sapiens, AAH02973.1), Necator (Necator americanus, ETN82101.1), Brugia (Brugia malayi, XP_001895788.1), Ascaris (Ascaris suum, ERG86215.1), and Zebra (Danio rerio, AAH76457.1).

cently, RNase H (EC 3.1.26.4) of Leishmania was investigated for understanding the function of it in this parasitic organism, which will lead to more effective and optimized chemotherapeutic drug-targeting strategies against the harmful afflictions it causes [10]. Therefore, we analyzed the DNA sequence of endoribonuclease from E. cinetorchis and characterized a recombinant endoribonuclease protein to enhance developing a new antisense oligonucleotide targeting to damage the parasites. In addition, we developed monoclonal antibodies to de- termine the location of endoribonuclease in adult $E$. cinetorchis and to obtain valuable information related to pathology and diagnosis.

Naturally infected Segmentina (polypylis) hemisphaerula were collected in Sinchang-myon, Asan-si, Chungnam Province, and then metacercariae were isolated. Five Sprague-Dawley rats (body weight: $120 \mathrm{~g}$ ) were infected with 50 metacercariae using a tuberculin syringe and maintained at $20^{\circ} \mathrm{C}$ in $50-55 \%$ humidity and a 12 hr-light/12 hr-dark cycle for 4 weeks. The 
presence of E. cinetorchis eggs were confirmed by microscopic examinations. Adult $E$. cinetorchis worms were isolated from the small intestine of a rat by dissection. To amplify the E. $c i$ netorchis endoribonuclease, degenerative primers described below were designed based on consensus sequences of Schistosoma haematobium (NCBI GenBank no. KGB32409.1), Schistosoma japonicum (AAW25442.1), Clonorchis sinensis (GAA56298.1), and Schistosoma mansoni (CCD59795.1). RT-PCR for amplification of the E. cinetorchis endoribonuclease was performed with the mRNA prepared above. The amplified product was inserted into a pGEM-T Easy vector (Promega, Madison, Wisconsin, USA). The sequence did not contain $5^{\prime}$ and $3^{\prime}$ ends; therefore, 3'-RACE was performed with a 3'-Full RACE Core Set (Takara Bio Inc., Otsu, Shiga, Japan) and specially designed primers: sense primer, 5'-YTN YTN GAY AAY TAY-3' (15 mers), and antisense primer, 5'-CTG ATC TAG AGG TAC CGG ATCC-3' (21 mers) ( $\mathrm{Y}=\mathrm{T}$ or $\mathrm{C}, \mathrm{N}=\mathrm{A}$ or $\mathrm{T}$ or $\mathrm{C}$ or $\mathrm{G}$ ). $5^{\prime}$-RACE was performed with a 5'-Full RACE Core Set (Takara Bio Inc.) and sense primer 5'-ATG ATT GCG TGG CCA ACA GTG-3' (21 mers) and antisense primer 5'- ATC GTT GTC AGC GCT CCA CC-3' (20 mers).

It was shown that the CDNA sequence E. cinetorchis endoribonuclease consists of 927 nucleotides, which encoded 308 amino acids, as determined by the DNASIS program. It is known that RNases $\mathrm{H}$ have an active site centered on a conserved motif composed of aspartate (D) and glutamate (E) residues, DEDD motif. Interestingly, we found putative DDEED motif instead of DEDD motif in parasites (Fig. 1A) [12].

The amino acid sequences showed 35\% similarity with that of S. haematobium, $35 \%$ with that of S. japonicum, $35 \%$ with that of S. mansoni, and $40 \%$ with that of C. sinensis, $6 \%$ with that of $M y$ cobacterium tuberculosis (AGQ35548.1), 7\% with that of Rattus norvegicus (AAH91209.1), 8\% with that of Mus musculus (AAH19411.1), 8\% with that of Homo sapiens (AAH02973.1), 5\% with that of Necator americanus (ETN82101.1), 6\% with that of Brugia malayi (XP_001895788.1), 5\% with that of Ascaris suum (ERG86215.1), and 5\% with that of Danio rerio (AAH76457.1), whereas endoribonucleases in S. haematobium, S. japonicum, and S. mansoni showed greater than $70 \%$ sequence similarity. Based on the low similarity of the E. cinetorchis ribonuclease with those of other parasites, it might have a unique character than other genes (Fig. 1A, B). Serine proteases, cysteine proteinases, and RNases of protozoans and helminths have been studies extensively. However, little is known about endoribonuclease in $S$. haematobium, S. japonicum, and S. mansoni. Therefore, we provided the first report on the molecular biological characteristics of

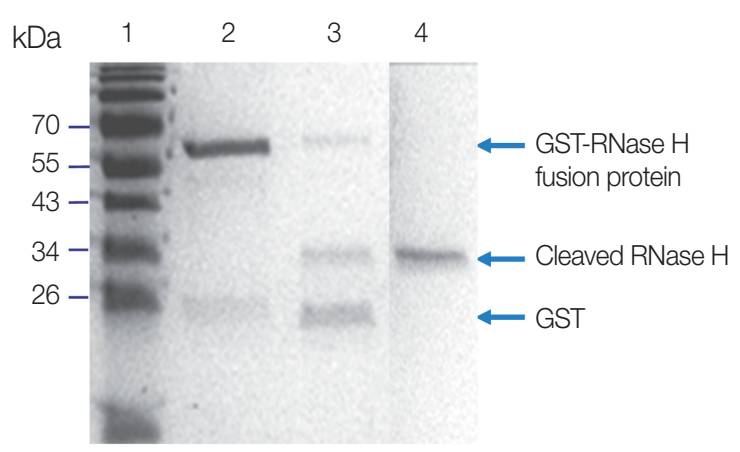

Fig. 2. Purification of recombinant $E$. cinetorchis endoribonuclease (ECRNH) from GST-fusion protein. Lane 1, molecular weight marker; lane 2, purified GST-fusion protein; lane 3, digested GSTfusion protein with thrombin 10 units; lane 4, recombinant EcRNH purified. For expression of EcRNH, the cDNA was inserted into pGEX-4T-1 (GST-tagged) plasmid vector and transformed into $E$. coli BL21 (DE3) plysS (Promega). Recombinant EcRNH was cleaved off from the fusion protein by digesting with thrombin (Sigma Co., St. Louis, Missouri, USA) and purified through a Sephacryl S-200 high resolution column chromatography (molecular weight: 1-80 kDa) (Pharmacia, Stockholm, Sweden).

the endoribonuclease in E. cinetorchis, including the full cDNA sequence.

To express a recombinant endoribonuclease protein in $E$. coli, an EcoRI (GAATTC) restriction site was added to the sense primer, 5'-GAATTC ATGATTGCGTGGCCAACAGTG-3' (27 mers), and a XhoI (CTCGAG) sequence was added to the antisense primer, 5'-CTCGAG ATCGTTGTCAGCGCTCCACC-3' (26 mers). The PCR product was inserted into a pGEM-T Easy vector (Promega) and transformed into E. coli DH5a for gene amplification. After confirming the DNA sequence of the coding region in pGEX-4T-1 (GST-tagged) expression vector, we obtained a transformed recombinant protein expressed clone, named E. coli BL21(DE3) plysS[pEndoEx]. The GST-tagged endoribonuclease protein was purified with GST-binding agarose resin (ELPIS-Biotech Inc., Daejeon, Korea) according to the manufacturer's manual. A soluble protein was successfully obtained, as confirmed by GST-specific antibody in a western blot analysis. The GST tag was removed by treatment with 1020 units thrombin (Fig. 2, lane 3) and cleaned with a Sephacyl S-200 High Resolution Gel (Fig. 2, lane 4). Based on results of the SDS-PAGE, the GST-free recombinant endoribonuclease protein showed a molecular weight of approximately $34 \mathrm{kDa}$ (Fig. 2, lane 4). Several expression vectors, pET29a (Histagged), pQE30 (His-tagged), and pBAD (His-tagged), and Escherichia coli M15 cell and BL21 (DE3) plysS as competent cells, were used in several combinations to express the cloned 

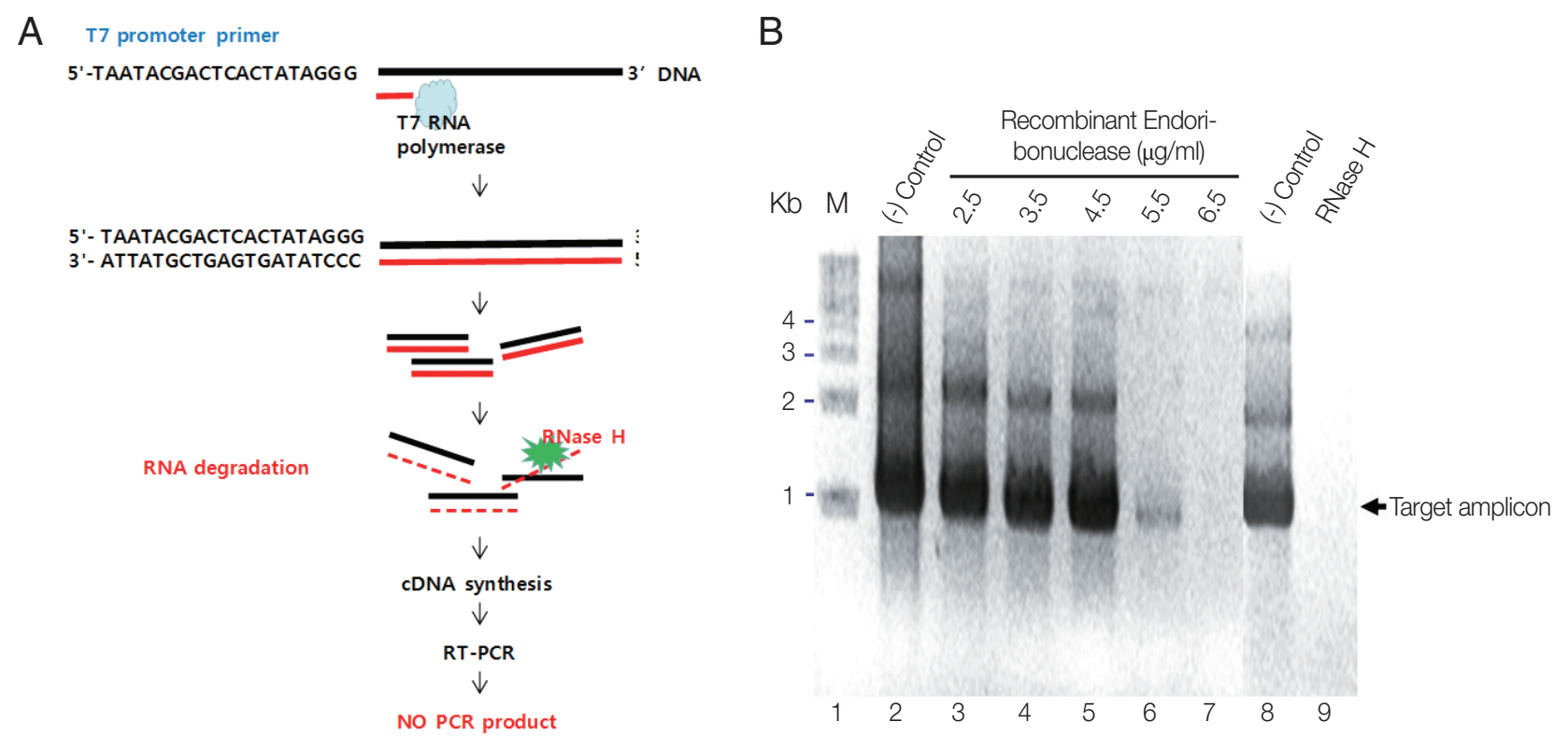

Fig. 3. (A) Diagram of activity analysis on endoribonuclease (RNase H). (B) Enzymatic activity of recombinant EcRNH. M, marker, 1-kb DNA ladder; (-) control, untreated with endoribonuclease; RNase H, treated with RNase H from Takara Co (60 units).

endoribonuclease gene; however, these attempts failed (data not shown). Even in several combinations, finding the best conditions to obtain the soluble protein was challenging. When E. coli BL21(DE3) plysS[pEndoEx] cells were cultured at $37^{\circ} \mathrm{C}$ with $0.5-1.0 \mathrm{mM}$ IPTG, a large quantity of insoluble protein (inclusion bodies) was expressed. Even in culture at $25^{\circ} \mathrm{C}$, inclusion bodies were observed (data not shown). Interestingly, small amounts of soluble proteins were obtained when the cells were cultured at $16^{\circ} \mathrm{C}$ for $24-48 \mathrm{hr}$.

To verify the enzymatic activity of recombinant endoribonuclease, Parniak's method was modified as described below [11]. To do this, first of all, we synthesized RNA from T7 promoter conjugated full cDNA of endonuclease using T7 RNA polymerase (Fig. 3A). Then, we treated them with various concentrations of endonuclease or RNase $\mathrm{H}$ to allowed RNA degradation. To see the various concentrations of amplified DNA according to their degraded RNA by the role of enzymes, RTPCR was done. One unit of endoribonuclease was defined as not produce the PCR product according to the character of RNase $H$. When adding purified recombinant endoribonuclease protein at different concentrations $(2.5,3.5,4.5,5.5$, and $6.5 \mu \mathrm{g}$ ) into the plasmid DNA, pEndo (the template) and the amplified PCR products showed the same amount according to the concentration of endoribonuclease (Fig. 3B, control lanes). Because the DNA was not the endoribonuclease sub- strate, it did not hydrolyze the DNA. However, by adding the DNA-RNA hybrid as a template for PCR, the amplified PCR products were reduced according to the endoribonuclease concentration. At an endoribonuclease concentration of 6.5 $\mu \mathrm{g}$, PCR product was not obtained (Fig. 3B, lane 7). Therefore, 1 unit of endoribonuclease was defined as $6.5 \mu \mathrm{g}$. When adding 1 unit of RNase H (Takara) to the PCR reaction, no PCR product was obtained (Fig. 3B, lane 9), similarly to the result with the recombinant endoribonuclease protein (Fig. 3B, lane 7). Based on these results, purified recombinant endoribonuclease protein was considered an RNase H. Therefore, modified enzymatic assay for RNase $\mathrm{H}$ activity may be useful to examine these kinds of enzymes based on PCR.

Generally, endoribonucleases contribute to mRNA stability, but the function of this endoribonuclease in cells is little known; for example, it has not yet been shown how this enzyme cuts the specific RNA site. Exceptionally, how Dicer and AGO2 recognize the substrates well? The intracellular location of endoribonucleases is very important to understanding the cleavage specificity of an endoribonuclease for dinucleotide sequences [9]. Biologically, RNase $\mathrm{H}$ appears to be involved in DNA replication and repair and possibly in transcription. By targeting antisense phosphorothioate oligodeoxynucleotides (ODN) to the essential mRNAs in Leishmania, the RNA-DNA hybrid-specific cleavage feature of RNase $\mathrm{H}$ can be manipulat- 
ed to operate in the host's advantage. The ablation of gene expression in the parasite can prevent its proliferation or lead to its death. Different classes of RNase $\mathrm{H}$ have been detected and characterized in several organisms ranging from bacteria to retroviruses to mammals, and it is interesting that they all share some homology at different levels. Theoretically, cotreatment of Leishmania with a possible activator of RNase $\mathrm{H}$ and an antisense oligonucleotide should accentuate the efficacy of the ODN and bring plausible chemotherapy treatment for leishmaniasis [10]. Therefore, our finding also contributes to developing new strategies based on antisense ODN to kill the parasites, including E. cinetorchis. However, we do not know yet why this protein located in the muscle layer only (data not shown); it may be expressed as essential proteins for parasite survival in this area. Therefore, it should be investigated in more details in the near future.

\section{ACKNOWLEDGMENTS}

We are grateful to members of the Public Health Centers in Asan City. This work was supported by the Inha University Research Fund.

\section{CONFLICT OF INTEREST}

We have no conflict of interest related to this study.

\section{REFERENCES}

1. Yamashita J. Echinostome. Progr Med Parasitol Japan 1964; 1:289-313

2. Ando R, Ozaki Y. On four new species of trematodes of the family Echinostomatidae. Dobutsugaku Zasshi 1923; 5: 108-119 (in Japanese).

3. Takahashi S, Ishii T, Ueno N. A human case of Echinostoma cinetorchis. Tokyo Iji Shinshi 1930; 2: 1326-1328 (in Japanese).

4. Takahashi S, Ishii T, Ueno N. The second human case of Echinostoma cinetorchis and a case of tapeworm in man. Tokyo Iji Shinshi 1930; 2: 1326-1327 (in Japanese).

5. Kawahara S, Yamamoto E. Human cases of Echinostoma cinetorchis. Tokyo Iji Shinshi 1933; 2: 1794-1796 (in Japanese).

6. Takahashi S. The life cycle of Echinostoma cinetorchis and Echinostoma macrorchis, particularly their first and second intermediate hosts. Fukuoka Ika Daigaku Zasshi 1927; 20: 712-723 (in Japanese).

7. Komiya Y. Metacercariae in Japan and adjacent territories. Section II. Special treatise. Progr Med Parasitol Japan 1965; 2: 1-328.

8. Seo BS, Cho SY, Hong ST, Hong SJ, Lee SH. Studies on parasitic helminths of Korea. 5. Survey on intestinal trematodes of house rats. Korean J Parasitol 1981; 19; 131-136.

9. Li WM, Barnes T, Lee CH. Endoribonucleases-enzymes gaining spotlight in mRNA metabolism. FEBS J 2010; 277: 627-641.

10. Bennett JR. Departments-leading the way-ribonuclease H: a tool in the battle against leishmaniasis. Chem Innov 2001; 31: 3-4.

11. Parniak MA, Min KL, Budihas SR, Le Grice SF, Beutler JA. A fluorescence-based high-throughput screening assay for inhibitors of human immunodeficiency virus-1 reverse transcriptase-associated ribonuclease H activity. Anal Biochem 2003; 322: 33-39.

12. Nowak E, Miller JT, Bona MK, Studnicka J, Szczepanowski RH, Jurkowski J, Le Grice SF, Nowotny M. Ty3 reverse transcriptase complexed with an RNA-DNA hybrid shows structural and functional asymmetry. Nat Struct Mol Biol 2014; 21: 389-396. 
